\title{
Quercetin Prevents Cardiac Hypertrophy Induced by Pressure Overload in Rats
}

\author{
Jing-Jun $\mathrm{HAN}^{1) * *}$, Jia $\mathrm{HAO}^{2) * *}$, Chan-Hyung $\mathrm{KIM}^{2)}$, Jang-Soo $\mathrm{HONG}^{3) *}$, Hee-Yul $\mathrm{AHN}^{2}$ * and Yong-Soon LEE ${ }^{4)}$ \\ ${ }^{1)}$ Department of Thoracic and Cardio vascular Surgery, Guang Dong Medical College FuTian Hospital, 3025 ShenNan Center Road, \\ ShenZhen, Guang-dong, China ${ }^{2}$ Departments of Pharmacology and ${ }^{3)}$ Thoracic and Cardiovascular Surgery, College of Medicine, \\ Chungbuk National University, Cheongju 361-763 and ${ }^{4)}$ Department of Veterinary Public Health, College of Veterinary Medicine, Seoul \\ National University, Seoul 151-742, Korea
}

(Received 25 December 2007/Accepted 2 February 2009)

\begin{abstract}
Inhibition of cardiac hypertrophy leads to a significant reduction in cardiovascular mortality and morbidity. Quercetin is by far the most abundant flavonoid and believed to ameliorate cardiovascular disease. Therefore, we investigated whether quercetin supplementation could attenuate the development of cardiac hypertrophy induced by pressure overload. Three weeks after suprarenal transverse abdominal aortic constriction, heart to body weight (HW/BW) ratio increased compared to the sham group $(3.40 \pm 0.06 \mathrm{mg} / \mathrm{g}$ versus $2.83 \pm 0.02 \mathrm{mg} / \mathrm{g}, P<0.001)$. The quercetin administered group showed complete inhibition of cardiac hypertrophy $(2.85 \pm 0.01 \mathrm{mg} / \mathrm{g}$, $P<0.001)$. Malonyldialdehyde production induced by pressure overload was suppressed by quercetin. The activities of extracellular signal-regulated kinase (ERK1/2), p38 MAP kinase, Akt and GSK-3 $\beta$ were significantly increased with pressure overload and attenuated by quercetin treatment. We conclude that quercetin appears to block the development of cardiac hypertrophy induced by pressure overload in rats and that these effects may be mediated through reduced oxidant status and inhibition of ERK1/2, p38 MAP kinase, Akt and GSK- $3 \beta$ activities.
\end{abstract}

KEY WORDS: cardiac hypertrophy, pressure overload, quercetin.

J. Vet. Med. Sci. 71(6): 737-743, 2009

Cardiac hypertrophy is a homeostatic response to elevated afterload that develops due to pressure or volume overload. Although cardiac hypertrophy is a compensatory response to increased wall stress in the early stage, it is followed by decompensation and ultimately leads to heart failure if the stimulus is sufficiently intense and prolonged.

Several intracellular signaling pathways including mitogen-activated protein kinase (MAPK), Janus kinase/signal transducers, activators of transcription (JAK/STAT), calcineurin [16], serine/threonine kinase, Akt and its downstream target, glycogen synthase kinase- $3 \beta$ (GSK-3 $\beta$ ) have been implicated in mechanical stress-induced cardiac hypertrophy. These observations have led to speculation that inhibition of these hypertrophic signals may be an effective means of clinical therapy in the treatment of pathological hypertrophy and heart failure.

Quercetin (3,3',4',5,7-pentahydroxyflavone) is a member of a group of naturally occurring compounds and is one of the most widely distributed bioflavonoids [5]. Several epidemiological studies have shown a significant inverse association between dietary flavonoids and long-term mortality from coronary heart disease. A very wide range of biological actions of flavonoids, including antioxidant, antiaggregant and vasodilatory effects, support the purported role

\footnotetext{
* Correspondence to: Prof. Dr. Ahn, H.-Y., Department of Pharmacology, College of Medicine, Chungbuk National University, Cheongju, 361-763, South Korea. e-mail: hyahn@chungbuk.ac.kr

Prof. Dr. HonG, J.-S., Department of Thoracic and Cardiovascular Surgery, College of Medicine, Chungbuk National University, Cheongju, 361-763, South Korea.

e-mail: jshong@chungbuk.ac.kr

**These authors contributed equally to this study
}

of quercetin protection against cardiovascular disease [4]. Recently, it has been reported that quercetin inhibits hypertrophy of cardiomyocytes induced by angiotensin II [15]. However, there is little information about the effect of quercetin on in vivo models of cardiac hypertrophy. Therefore, the purpose of this study is to investigate whether chronic administration of an oral daily dose of quercetin $(5 \mathrm{mg}$ or 10 $\mathrm{mg}$ /day) can attenuate cardiac hypertrophy induced by pressure overload and to elucidate its potential mechanisms of action.

\section{MATERIALS AND METHODS}

In vivo hypertrophy model: Male Sprague Dawley rats (7 weeks old, 200 220 g) were purchased from Koatech (Pyoungtaek, Korea). The experimental protocol was approved by Chungbuk National University Medical School Research Institutional Animal Care and Use Committee. All surgical procedures were performed with the animals under anesthetized with ketamine $(80 \mathrm{mg} / \mathrm{kg} \mathrm{IP})$ and xylazine $(5 \mathrm{mg} / \mathrm{kg} \mathrm{IP})$. Transaortic constriction was created using a 4-0 suture tied twice around the suprarenal aorta and a 21-gauge needle. The needle was then removed yielding a $0.8 \mathrm{~mm}$ internal diameter. Sham operated rats were randomly assigned and underwent the same procedure, with the exception that the aorta was not constricted. A freshly prepared solution with different doses of quercetin (Sigma, St Louis, MO, U.S.A.) was supplied every day to aortic banded or sham operated rats as the sole source of drinking water over a period of 21 consecutive days, whereas control animals were supplied with water from the same source, lacking quercetin. 
Quercetin administration

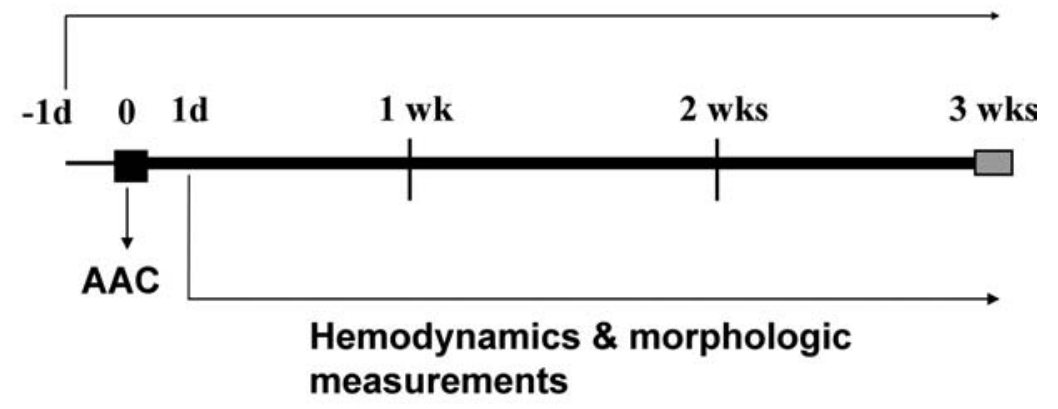

Fig. 1. Experimental design. One day before the operation, rats were randomly treated with quercetin or no drug. Quercetin was dissolved in drinking water, and the administered solutions were replaced every day for 3 weeks. Hemodynamic and morphologic measurements were done at 1 day, 1 week, 2 and 3 weeks after abdominal aortic constriction (AAC).

Hemodynamics measurements: The right carotid and left femoral arteries were cannulated with 20-gauge intravenous cannulas. Mean arterial blood pressure, heart rate and pressure gradient between carotid and femoral arteries were obtained from a pressure transducer attached to each cannula, which was inserted through a fluid-filled catheter. They were recorded using a computer data acquisition system (ADinstrument, model ML870, NSW, Australia), after blood pressure was stable for $10 \mathrm{~min}$.

Assessment of lipid peroxidation: Lipid peroxidation in cardiac tissues was measured by a modified thiobarbituric acid method [19]. Heart homogenates were centrifuged at $2,000 \times \mathrm{g}$ for $10 \mathrm{~min}$. Supernatants $(0.5 \mathrm{~mL})$ were mixed with $1.5 \mathrm{~mL}$ and glacial acetic acid $(30 \% \mathrm{w} / \mathrm{v}), 1 \mathrm{~mL}$ thiobarbituric acid $(0.7 \% \mathrm{w} / \mathrm{v})$ and $0.5 \mathrm{~mL}$ water, followed by boiling for $60 \mathrm{~min}$ and then cooled to room temperature. After the addition of 2 volumes of a butanol-pyridine mixture $(15: 1, \mathrm{v} / \mathrm{v})$, the reaction mixture was shaked vigorously and centrifuged at 3,000 g for $20 \mathrm{~min}$. The butanol extract was used for malondialdehyde (MDA) assay with a spectrophotometer at $532 \mathrm{~nm}$. The amount of MDA was calculated by the molar extinction coefficient of $1.52 \times 10^{5} / \mathrm{M} / \mathrm{cm}$.

Western blot analysis: Heart lysates $(15 \mu \mathrm{g})$ were subjected to SDS-PAGE as described previously [9]. The blots were probed with specific antibodies (Cell Signaling Technology, Beverly, MA, U.S.A., 1:100), and the immune complex was visualized with the ECL plus system (Santa Cruz Biotechnology, Santa Cruz, CA, U.S.A.).

C-Jun N-terminal kinase activity assay: The activity of cJun N-terminal kinase (JNK) was measured as described previously [21].

Statistical analysis: Results are presented as mean \pm SEM. Data obtained were compared using the unpaired Students $t$-test or one-way ANOVA. Statistical significance was defined as a value of $P<0.05$.

\section{RESULTS}

Effects of quercetin on heart and liver morphology in pressure overload rats: The amount of water intake was measured every day. Administration of quercetin in the drinking water at $5 \mathrm{mg}$ or $10 \mathrm{mg} /$ head/day did not alter water intake in either the sham operated or aortic constricted rats. After 3 weeks of aortic constriction, heart weight to body weight ratio (HW/BW) was increased by $23 \%$ compared with sham operated rats. Quercetin administration significantly attenuated this increase in a dose-dependent manner (Fig. 2A and 2B, $P<0.001$ ). Inhibitory effects of quercetin were rapidly shown and sustained during experimental period (Fig. 4).

Liver to body weight ratios ( $\mathrm{LW} / \mathrm{BW}$ ) were no significant changes with pressure overload (Fig. $2 \mathrm{C}, P>0.05$ ). Neither surgical operation nor quercetin has a significant effect on normal rat heart or liver (Fig. 3A and 3B).

Effects of quercetin on blood pressure and heart rate in pressure overload rats: Aortic banding produced a large pressure gradient between the carotid and femoral arteries (Table $1, P<0.001$ ). Treatment with $10 \mathrm{mg}$ quercetin shows no significant difference in pressure gradient compared with aortic-banded group. There was also no significant change in heart rates.

Effects of quercetin on the oxidative stress in pressure overload rats: Reactive oxygen species (ROS) have been shown to play a key role in myocardial hypertrophy induced by pressure overload. MDA is a product of cell membrane lipid peroxidation, therefore, a direct marker of oxidative cell toxicity. As shown in Fig. 5, myocardial MDA production was significantly increased in aortic banded rats than sham operated rats. This increased membrane lipoperoxidation was abated after treatment of quercetin with a dosedependent manner. Quercetin alone treatment had no significant changes among them.

Effects of quercetin on pressure overload-induced ERK1/ 2, p38 MAPK, Akt and GSK-3 $\beta$ activation in rats: We next examined the protein expression and phosphorylation activity of Akt, GSK-3 $\beta$, and MAPK families including ERK1/2, p38 MAPK, and JNK1/2 in the hearts subjected to pressure overload (Fig. 6). The total protein expressions of MAPK 
A
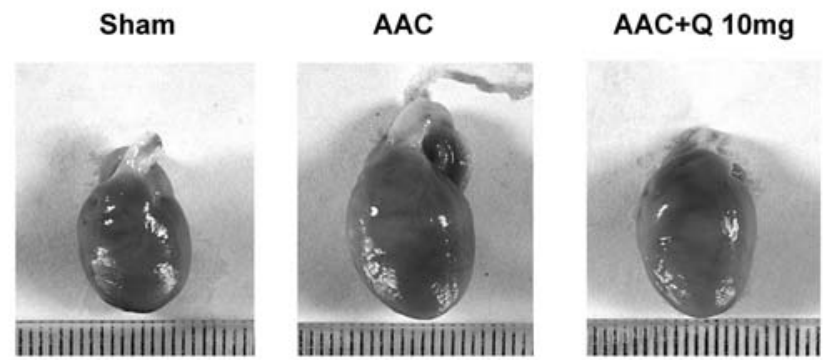

B

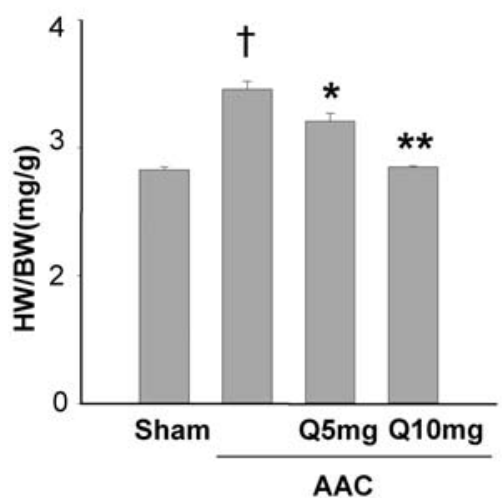

C

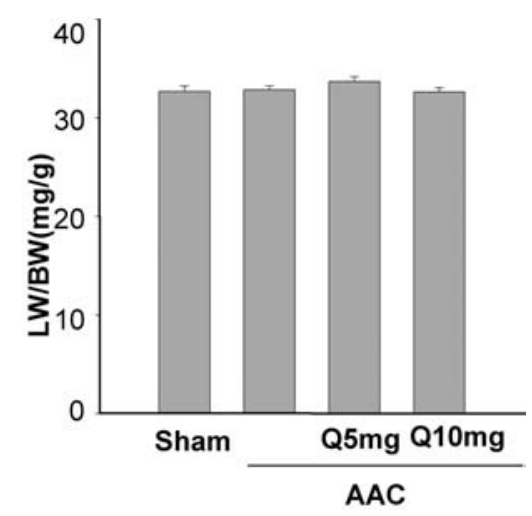

Fig. 2. Inhibition of pressure overload-induced cardiac hypertrophy in vivo by quercetin. A: Representative hearts of rats treated with abdominal aortic constriction (AAC) or AAC+Q10 $\mathrm{mg}$ for 3 weeks (Scale bar=1 mm). B: Heart wet weight was normalized to body weight (HW/BW, mg/g) in each rat as an index of cardiac hypertrophy. Heart weight to body weight ratio was attenuated in a dose-dependent manner by quercetin 3 weeks after AAC surgery. ${ }^{\dagger} P<0.001$ vs. Sham; ${ }^{*} P<0.001$ vs. AAC group, $(\mathrm{n} \geq 11)$. C: Liver weight to body weight ratios $(\mathrm{LW} / \mathrm{BW}, \mathrm{mg} / \mathrm{g})$. AAC operation or quercetin administration has no significant effects on the liver in rats $(\mathrm{n} \geq 11)$.

A

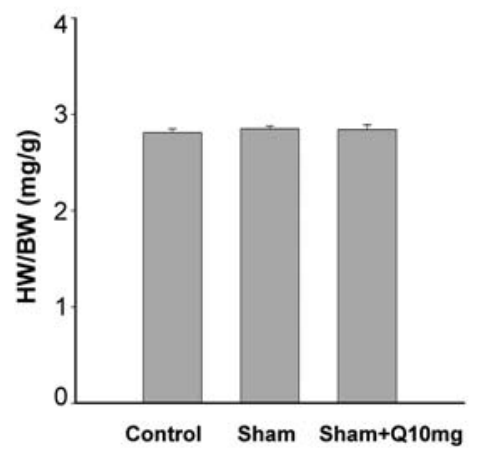

B

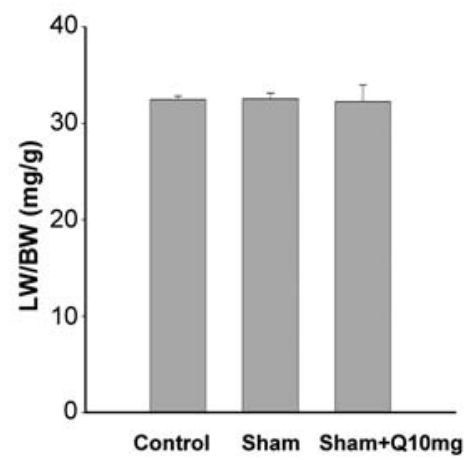

Fig. 3. Effects of sham operation or quercetin administration for 3 weeks on normal heart (A) and liver (B). Sham operation or quercetin has no significant effects on normal heart and liver weight $(n=7)$.

families, Akt and GSK- $3 \beta$ were not changed in pressureoverloaded hearts. By pressure overload, ERK1/2, p38 MAPK, Akt, and GSK- $3 \beta$ demonstrated a biphasic pattern of activation and significant activation of the phosphoryla- tion was first observed at $30,30,30$, and $15 \mathrm{~min}$, respectively, and sustained from $6 \mathrm{hr}$ to 3 weeks after aortic constriction. No remarkable increase in the phosphorylation of JNK $1 / 2$ was found throughout the experimental period. 


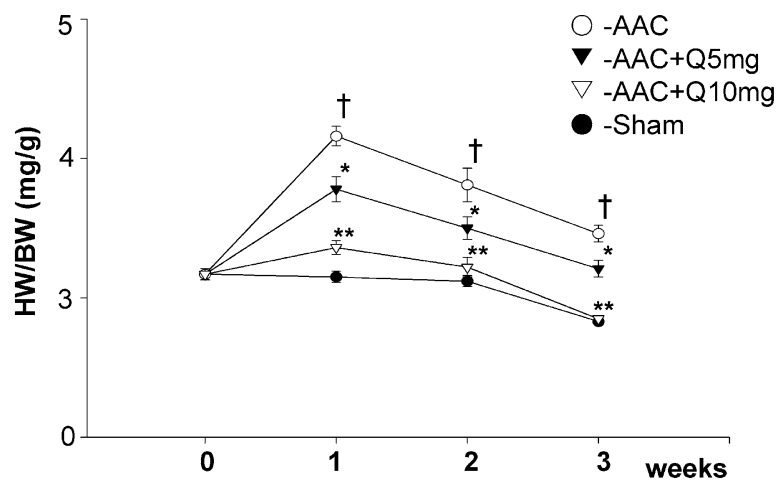

Fig. 4. Inhibition of cardiac hypertrophy by quercetin for the different time intervals with pressure overload in rats. Quercetin inhibited pressure overload-induced cardiac hypertrophy from 1 week in a dose-dependent manner. ${ }^{\dagger} P<0.001$ vs. Sham groups. ${ }^{*} P<0.01,{ }^{* *} P<0.001$ vs. Abdominal aortic constricted (AAC) rats $(\mathrm{n} \geq 11)$.

Pressure overload increased phosphorylation of ERK1/2, p38 MAPK, Akt, and GSK-3 $\beta$ soon after aortic banding prior to hypertrophic changes. Therefore, we examined the effects of quercetin on the pressure overload-induced ERK1/2, p38 MAPK, Akt, and GSK-3 $\beta$ phosphorylation. As shown in Fig. 7, phosphorylation of ERK1/2, p38 MAP kinase, Akt and GSK- $3 \beta$ induced by aortic constriction was significantly reduced by quercetin in a dose-dependent manner.

\section{DISCUSSION}

Cardiac hypertrophy leads to congestive heart failure, a major cause of death worldwide. Epidemiological studies suggest that increased dietary intake of flavonoids is associated with a reduced risk of cardiovascular diseases. Quercetin is, the most abundant flavonoid and is found in many fruits and vegetables. Various pharmacological activities of quercetin have been demonstrated including antioxidation by scavenging free radicals and the prevention of atherosclerosis and chronic inflammation [2]. Furthermore, it has

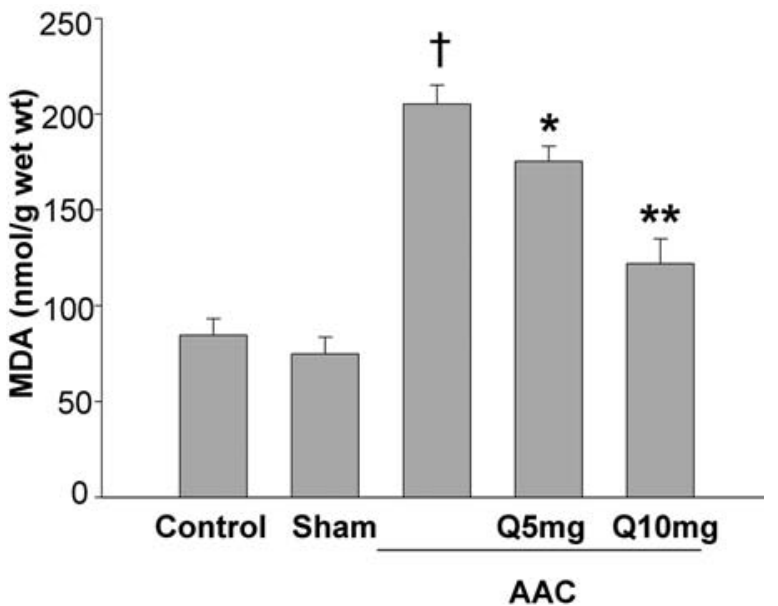

Fig. 5. Effects ofquercetinonpressureoverload-inducedlipidperoxidation. Compared with the sham-operated hearts, thepressureoverloaded hearts show asignificantincreasein MDAproduction. This effect is blunted by quercetin treatment. ${ }^{\dagger} P<0.001 \mathrm{vs}$. Sham; ${ }^{*} P<0.001$ vs. AAC group, $(\mathrm{n} \geq 4)$.

been demonstrated that quercetin suppresses tumor growth and has an antiangiogenic effect [17]. Cell proliferation and angiogenic mechanisms are known to contribute to the regulation of cardiac hypertrophy. Therefore, we examined the effect of quercetin on pressure overload induced cardiac hypertrophy. In the present study, we found that an oral daily dose of the bioflavonoid can function to attenuate cardiac hypertrophic changes induced by pressure overload.

Sustained high blood pressure is one of the most powerful causes of the development of cardiac hypertrophy. Quercetin and related bioflavonoids have shown vasodilatory effects in an isolated aorta stimulated with noradrenaline, $\mathrm{KCl}$ or phorbol esters [13]. Likewise, it has been demonstrated that quercetin has anti-hypertensive effects on spontaneously hypertensive rats [3]. Therefore, blockage of pressure overload-induced cardiac hypertrophy by quercetin raised the possibility that quercetin might ameliorate the hypertension induced by aortic banding and that the blockage of hypertrophy might have been secondary to afterload

Table 1. Effects of quercetin on blood pressure and heart rate in pressure-overloaded rats

\begin{tabular}{|c|c|c|c|c|c|c|}
\hline & & $\begin{array}{c}\mathrm{ASP} \\
(\mathrm{mmHg})\end{array}$ & $\begin{array}{l}\text { CADP } \\
(\mathrm{mmHg})\end{array}$ & $\begin{array}{c}\text { MAP } \\
(\mathrm{mmHg})\end{array}$ & $\begin{array}{l}\text { Gradient } \\
(\mathrm{mmHg})\end{array}$ & $\begin{array}{c}\text { HR } \\
\text { (beat } / \text { min) }\end{array}$ \\
\hline \multirow{4}{*}{1 day } & Sham $(n=3)$ & $138 \pm 4$ & $113 \pm 4$ & $122 \pm 4$ & $15 \pm 2$ & $337 \pm 9$ \\
\hline & $\mathrm{AAC}(\mathrm{n}=4)$ & $155 \pm 7$ & $126 \pm 4$ & $136 \pm 5$ & $23 \pm 3$ & $330 \pm 8$ \\
\hline & $\mathrm{AAC}+\mathrm{Q} 10 \mathrm{mg}(\mathrm{n}=3)$ & $152 \pm 7$ & $122 \pm 9$ & $132 \pm 8$ & $17 \pm 8$ & $323 \pm 14$ \\
\hline & Sham $(n=3)$ & $160 \pm 3$ & $130 \pm 3$ & $140 \pm 2$ & $11 \pm 4$ & $310 \pm 6$ \\
\hline \multirow[t]{3}{*}{1 week } & $\operatorname{AAC}(\mathrm{n}=4)$ & $198 \pm 5$ & $168 \pm 3$ & $178 \pm 4$ & $36 \pm 1^{\dagger}$ & $330 \pm 9$ \\
\hline & $\mathrm{AAC}+\mathrm{Q} 10 \mathrm{mg}(\mathrm{n}=3)$ & $195 \pm 6$ & $163 \pm 11$ & $174 \pm 9$ & $39 \pm 2 * *$ & $333 \pm 9$ \\
\hline & Sham $(n=6)$ & $169 \pm 3$ & $134 \pm 4$ & $146 \pm 4$ & $10 \pm 3$ & $320 \pm 3$ \\
\hline \multirow[t]{2}{*}{3 weeks } & $\mathrm{AAC}(\mathrm{n}=4)$ & $218 \pm 8$ & $184 \pm 7$ & $195 \pm 7$ & $45 \pm 2^{\dagger}$ & $308 \pm 8$ \\
\hline & $\mathrm{AAC}+\mathrm{Q} 10 \mathrm{mg}(\mathrm{n}=4)$ & $205 \pm 4$ & $174 \pm 5$ & $184 \pm 5$ & $45 \pm 7 * *$ & $315 \pm 13$ \\
\hline
\end{tabular}

Values are means \pm SEM. AAC, abdominal aortic constriction; CASP, carotid artery systolic pressure; CADP, carotid artery diastolic pressure; MAP, mean arterial pressure; Gradient, gradient between carotid and femoral artery pressures; HR, heart rate. ${ }^{\dagger} P<0.001$ or $* * P<0.001 v s$. Sham group. 


\section{Phospho-ERK1/2}

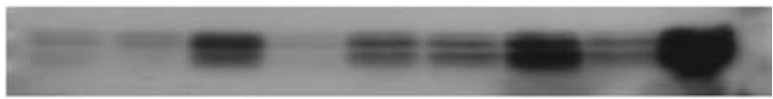

ERK1/2

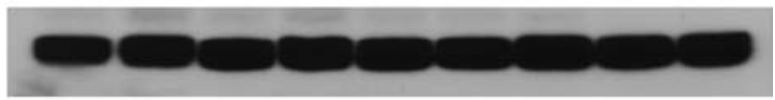

Phospho- p38

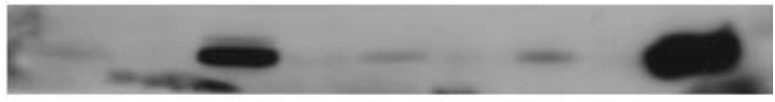

p38

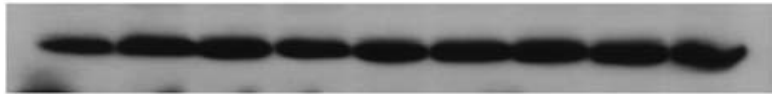

Phospho- c-Jun

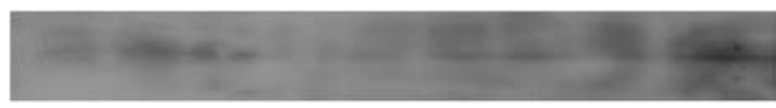

Phospho-Akt

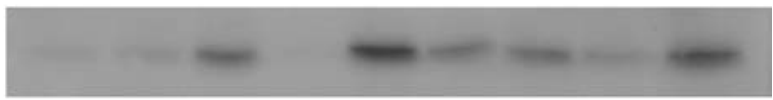

Akt

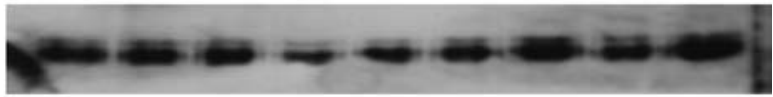

Phospho-GSK3 $\beta$

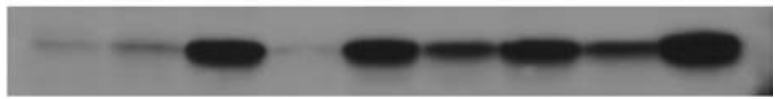

GSK3 $\beta$

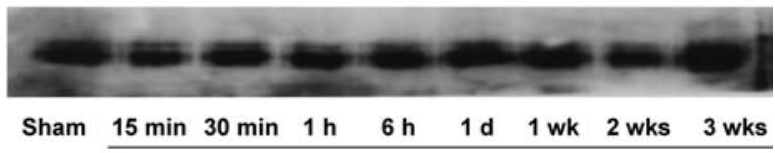

AAC

Fig. 6. Pressure overload-induced activation of ERK1/2, p38 MAP kinase, Akt and GSK$3 \beta$ in rats. The activities of ERK1/2, p38 MAP kinase, Akt and GSK- $3 \beta$ were measured by Western blot analysis with a phospho-specific antibody. JNK activity was measured by Western blot analysis with a phospho-specific c-Jun antibody. The data presented are representative of a minimum of three separate experiments.

\section{$30 \mathrm{~min}$}

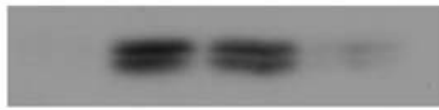

Phospho-p38

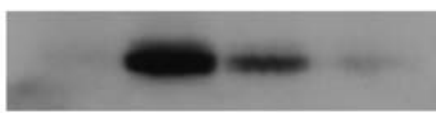

Phospho-Akt

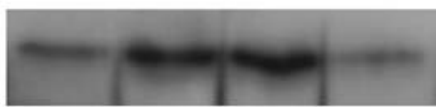

Phospho-GSK3 $\beta$

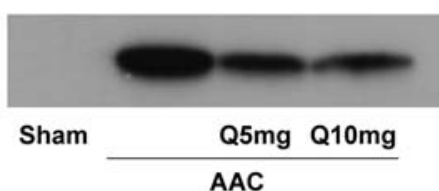

1 wk
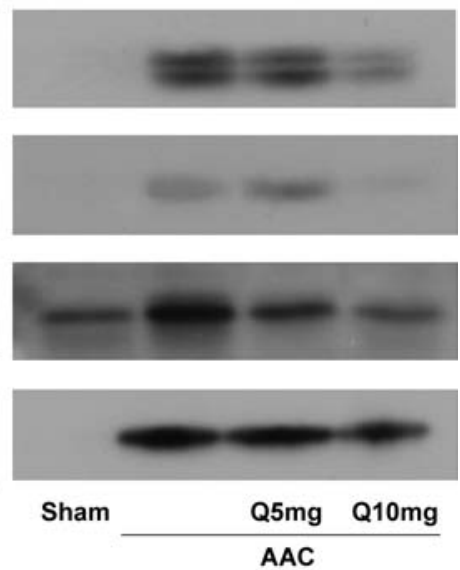

Fig. 7. Effects of quercetin on pressure overload-induced activation of ERK1/2, p38 MAP kinase, Akt and GSK-3 $\beta$. Quercetin inhibited phosphorylation activities of ERK1/2, p38 MAP kinase, Akt and GSK- $3 \beta$ in a dose-dependent manner. The data presented are representative of a minimum of three separate experiments. 
reduction rather than by direct cardiac effects. In this study, aortic constriction produced a large pressure gradient between carotid and femoral arteries, which was increased to a similar magnitude in the quercetin treated aortic constricted animals (Table 1). Measurements at different times after surgery revealed that quercetin had no significant effect on systemic blood pressure, after aortic banding. By contrast, it has been recently reported that quercetin supplemented diets lower blood pressure to attenuate cardiac hypertrophy with aortic constriction [8]. The conflicting results between this study and Jalili et al. are probably due to the difference in the dose of quercetin administered. The dose of quercetin used in this study is $\approx 3$ times lower than the dose used by Jalili et al. $(130 \mathrm{mg} / \mathrm{kg})$. Although the beneficial effect of quercetin on cardiovascular function seems to be related to its blood pressure lowering effect, it is unlikely that reduction in cardiac hypertrophy by this low dose of quercetin was caused by relief of afterload of hearts. Our data suggest that the primary target organ of quercetin is the heart rather than blood vessels and that its effects on ventricular weight may be attributed to attenuation of signal transduction pathways that regulate cardiac hypertrophy. In this study, we examined the time course of hypertrophic change after aortic constriction and showed that cardiac hypertrophy was observed as early as 1 week and quercetin inhibited this early hypertrophic change induced by pressure overload (Fig. 4). Moreover, aortic banding resulted in a peak increase in $\mathrm{HW} / \mathrm{BW}$ ratio at 1 week after pressure overload, and gradually decreased as time goes on. Therefore, we investigated the inhibitory effect of quercetin on hypertophic signal transduction at $30 \mathrm{~min}$ and 1 week after aortic constriction (Fig. 7).

Numerous studies have shown that MAPKs are strongly activated by ROS, which are known to be produced by pressure overload. Moreover, a growing body of evidence has suggested that MAPKs play important roles in cardiac hypertrophy [16]. This indicates that blocking MAPKs pathways might be an important mechanism that could account for the protective effect of quercetin. In the present study, the activities of ERK1/2, p38, Akt and GSK-3 $\beta$, but not JNK, were significantly increased after aortic banding prior to morphological changes and were attenuated by quercetin treatment, in a dose-dependent manner. By contrast, previous study reported that phosphorylations of ERK $1 / 2$ and Akt were not affected by quercetin treatment. This discrepancy may be explained by more severe banding conditions used by Jalili et al. [8]. On the other hand, GSK$3 \beta$ was not phosphorylated in response to Akt activation. Although GSK- $3 \beta$ is an established target of Akt, it is likely that regulation of GSK- $3 \beta$ is not solely dependent on Akt in pressure overloaded rat heart. Possible upstream protein kinases that regulate GSK- $3 \beta$ are integrin-linked kinase, protein kinase $\mathrm{A}$, protein kinase $\mathrm{C} \delta$, protein kinase $\mathrm{C} \zeta \mathrm{p} 90$ ribosomal S6 kinase, and $\mathrm{p} 70^{\mathrm{S} 6 \mathrm{~K}}$ [7]. In the present study, we examined the effect of quercetin on a number of downstream signaling molecules that regulate cardiac hypertrophy. However, it remains possible that the effects that we observed on cardiac hypertrophy are produced by inhibition of other signal pathway or an alternative cellular function of quercetin. Additional work will be necessary to investigate the precise mechanisms of quercetin.

ROS have been shown to act as intracellular signaling molecules in stress response, in a variety of cell types, leading to apoptosis, proliferation, and transformation. In cardiomyocytes, ROS have been found to mediate cardiac hypertrophy induced by several stimuli, such as mechanical stretch [14], angiotensin II, and tumor necrosis factor- $\alpha$ (TNF- $\alpha$ ). Quercetin has been known to have antioxidant activity. In vitro, quercetin inhibits hypoxanthine-xanthine oxidase activity and scavenges superoxide, and hydroxyl radicals. After oral administration, quercetin metabolites retain the antioxidant properties of the parent compound [11]. We found that the levels of MDA were markedly increased in the abdominal aortic constriction group, and quercetin treatment resulted in elimination of the increase in MDA. Therefore, we propose that the antioxidant effect might play a role in the prevention of cardiac hypertrophy in quercetin treated rats.

Different location of aortic constriction may differently activate the signal transduction pathway. When the aortic arch was constricted, so mechanical force may have been the major cause of cardiac hypertrophy. In contrast, reninangiotensin system (RAS) may be involved predominantly in the suprarenal abdominal aorta constriction and contribute to the initial development of cardiac hypertrophy and sympathetic activation in the compensated heart. It has been shown that losartan, an angiotensin $\mathrm{II}_{\mathrm{AT}}$ receptor antagonist, attenuates cardiac hypertrophy in suprarenal abdominal aorta constricted rat [10], but has no effect on the weight gain of the ventricle during aortic arch constriction [1]. Given that quercetin can inhibit not only angiotensin converting enzyme activity [6] but also angiotensin II-mediated signal transduction in cultured cardiomyocytes [15], the antihypertrophic effect of quercetin in the present study is possible to be mediated by RAS blockage. However, quercetin had no significant effect on systemic blood pressure that is sensitive to angiotensin II. These findings suggest that inhibition of RAS by quercetin, if any, does not play a major role in quercetin-mediated antihypertrophic effect.

Previous reports that quercetin induced apoptosis of tumor cells raise the possibility that apoptosis in cardiomyocytes may occur in our animal model, leading to a reduction in cardiac mass. However, it has been previously reported that pressure overload, itself, leads to an increase in TUNEL-positive cardiomyocytes [20]. Moreover, quercetin does not significantly affect the survival of cultured rat cardiomyocytes and shows a protective effect against chemotherapy-induced cardiotoxicity [12]. Although quercetin has proapoptotic effects in transformed cells, apoptosis of cardiomyocytes does not appear to be responsible for the reduction of heart mass.

Calcineurin inhibitors, such as cyclosporine A and FK506, have been well known to inhibit cardiac hypertrophy. However, nephrotoxicity and the immunosuppressive 
effect of calcineurin inhibitors limit their therapeutic benefit [18]. In our experiment, quercetin completely inhibited cardiac hypertrophy induced by pressure overload without significant effects on normal heart and liver (Fig. 3 A and B). There was no evidence of nonspecific toxicity, since the effects on normal growth, weight gain, or physical activity was not found. Moreover, quercetin is one of the most widely distributed bioflavonoids, which are naturally occurring compounds, found in regularly consumed food. Therefore, quercetin, or its derivatives, might be a useful medicine to suppress cardiac hypertrophy with low toxicity. Future studies are necessary to examine whether quercetin can regress established cardiac hypertrophy, which might be of greater clinical significance, because the patient would most likely already have hypertrophy at the initiation of treatment.

ACKNOWLEDGEMENT. This work was supported by the research grants of the Chungbuk National University in 2007.

\section{REFERENCES}

1. Baba, H. A., Iwai, T., Bauer, M., Irlbeck, M., Schmid, K. W. and Zimmer, H. G. 1999. Differential effects of angiotensin II receptor blockade on pressure-induced left ventricular hypertrophy and fibrosis in rats. J. Mol. Cell. Cardiol. 31: 445-455.

2. Corvazier, E. and Maclouf, J. 1985. Interference of some flavonoids and nonsteroidal anti-inflammatory drugs with oxidative metabolism of arachidonic acid by human platelets and neutrophils. Biochem. Biophys. Acta. 835: 315-321.

3. Duarte, J., Perez-Palencia, R., Vargas, F., Ocete, M. A., PerezVizcaino, F., Zarzuelo, A. and Tamargo, J. 2001. Antihypertensive effects of the flavonoid quercetin in spontaneously hypertensive rats. Br. J. Pharmacol. 133: 117-124.

4. Duarte, J., Perez-Vizcaino, F., Utrrilla, M. P., Jimeanez, J., Tamargo, J. and Zarzuelo, A. 1993. Vasodilatory effects of flavonoids in rat aortic smooth muscle. Structure-activity relationships. Gen. Pharmacol. 24: 857-864.

5. Formica, J. V. and Regelson, W. 1995. Review of the biology of quercetin and related bioflavonoids. Food Chem. Toxicol. 33: $1061-1080$.

6. Hackl, L. P., Cuttle, G., Dovichi, S. S., Lima-Landman, M. T. and Nicolau, M. 2002. Inhibition of angiotesin-converting enzyme by quercetin alters the vascular response to brandykinin and angiotensin I. Pharmacology 65: 182-186.

7. Hardt, S. E. and Sadoshima, J. 2002. Glycogen synthase kinase-3 $\beta$. A novel regulator of cardiac hypertrophy and development. Circ. Res. 90: 1055-1063.

8. Jalili, T., Carlstrom, J., Kim, S., Freeman, D., Jin, H., Wu, T. C., Litwin, S. E. and David, S. J. 2006. Quercetin-supplemented diets lower blood pressure and attenuate cardiac hypertrophy in rats with aortic constriction. J. Cardiovasc. Pharmacol. 47: 531-541.
9. Kim, C. H., Cho, Y. S., Chun, Y. S., Park, J. W. and Kim, M. S. 2002. Early expression of myocardial HIF-1alpha in response to mechanical stresses: regulation by stretch-activated channels and the phosphatidylinositol 3-kinase signaling pathway. Circ. Res. 90: E25-33.

10. Li, J., Li, P., Feng, X., Li, Z., Hou, R., Han, C. and Zhang, Y. 2003. Effects of losartan on pressure overload-induced cardiac gene expression profiling in rats. Clin. Exp. Pharmacol. Physiol. 30: 827-832.

11. Manach, C., Morand, C., Crespy, V., Demigne, C., Texier, O., Regerat, F. and Remesy, C. 1998. Quercetin is recovered in human plasma as conjugated derivatives which retain antioxidant properties. FEBS Lett. 26: 331-336.

12. Mojzisova, G., Mirossay, L., Kucerova, D., Kyselovic, J., Mirossay, A. and Mojzis, J. 2006. Protective effect of selected flavonoids on in vitro daunorubicin-induced cardiotoxicity. Phytother. Res. 20: 110-114.

13. Perez-Vizcaino, F., Utrilla, M. P., Jimenez, J., Tamargo, J. and Zarzuelo, A. 1993. Vasodilatory effects of flavonoids in rat aortic smooth muscle. Structure-activity relationships. Gen. Pharmacol. 24: 857-864.

14. Pimentel, D. R., Amin, J. K., Xiao, L., Miller, T., Viereck, J., Oliver-Krasinski, J., Baliga, R., Wang, J., Siwik, D. A., Singh, K., Pagano, P., Colucci, W. S. and Sawyer, D. B. 2001. Reactive oxygen species mediate amplitude-dependent hypertrophic and apoptotic responses to mechanical stretch in cardiac myocytes. Circ. Res. 89: 453-460.

15. Qin, T. C., Chen, L., Yu, L. X. and Gu, Z. L. 2001. Inhibitory effect of quercetin on cultured neonatal rat cardiomyocytes hypertrophy induced by angiotensin. Acta Pharmacol. Sin. 22: 1103-1106.

16. Ruwhof, C. and Laarse, A.V.D. 2000. Mechanical stressinduced cardiac hypertrophy: mechanisms and signal transduction pathways. Cardiovasc. Res. 47: 23-37.

17. Tan, W. F., Lin, L. P., Li, M. H., Zhang, Y. X., Tong, Y. G., Xiao, D. and Ding, J. 2003. Quercetin, a dietary-derived flavonoid, possesses antiangiogenic potential. Eur. J. Pharmacol. 459: $255-262$.

18. Ventura, H. O., Malik, F. S., Mehra, M. R., Stapleton, D. D. and Smart, F. W. 1997. Mechanisms of hypertension in cardiac transplantation and the role of cyclosporine. Curr. Opin. Cardiol. 12: 375-381.

19. Yagi. K. 1982. pp. 223-242. Lipid Peroxides in Biology and Medicine.Academic Press, New York.

20. Yamaguchi, O., Higuchi, Y., Hirotani, S., Kashiwase, K., Nakayama, H., Hikoso, S., Takeda, T., Watanabe, T., Asahi, M., Taniike, M., Matsumura, Y., Tsujimoto, I., Hongo, K., Kusakari, Y., Kurihara, S., Nishida, K., Ichijo, H., Hori, M. and Otsu, K. 2003. Targeted deletion of apoptosis signal-regulating kinase 1 attenuates left ventricular remodeling. Proc. Natl. Acad. Sci. U.S.A. 100: 15883-15888.

21. Zheng, Y., Song, H. J., Kim, C. H., Kim, H. S., Kim, E. G., Sachinidis, A. and Ahn, H. Y. 2004. Inhibitory effect of epigallocatechin 3-O-gallate on vascular smooth muscle cell hypertrophy induced by angiotensin II. J. Cardiovasc. Pharmacol. 43: 200-208. 